\section{CARDIAC TROPONIN I AND NATRIURETIC PEPTIDE NT-PROBNP IN CHILDREN WITH OPERATED CONGENITAL CARDIAC MALFORMATIONS}

A. Butnariu1 ${ }^{1}$, L. Dican², M. Chira², G. Samasca², M. Marc ${ }^{2}$, M. Andreica ${ }^{2}$

${ }^{1}$ Pediatrics, University of Medicine and Pharmacy 'Iuliu Hatieganu' Cluj, '2University of Medicine and Pharmacy Iuliu Hatieganu Cluj, Cluj-Napoca, Romania

Background and aim: Cardiac troponin I (cTnl) and natriuretic peptide NT-proBNP are useful biomarkers in the evaluation of the sick heart. We aimed to detect a potential early postoperative myocardial disease in children with congenital cardiac malformations undergoing heart surgery.

Methods: 24 patients aged between 6 months and 3 years with operated cyanogenic cardiac malformations and 17 control subjects were studied. cTnl was determined using an immunochemical method with detection by Immulite electrochemiluminescence (ECLIA); reference values $<0.2 \mathrm{ng} / \mathrm{ml}$. Serum NT-proBNP levels were assessed by the ELISA immunoenzymatic method (Enzyme-linked Immunosorbent Assay) using a standardized kit containing monoclonal antibodies that recognize epitopes located in the NT-proBNP portion. Serum was taken from patients within 24 hours post-operation.

Results: The mean cTnl value in patients with congenital heart diseases was $10.23 \mathrm{ng} / \mathrm{ml}$, compared to those of the controls situated below the detection limit $<0.2 \mathrm{ng} / \mathrm{ml}$. 50-fold higher cTnl values were found in patients compared to healthy subjects, suggesting myocardial injury. The mean value of NT-proBNP was $30.42 \mathrm{fmol} / \mathrm{ml}$.

There was a positive correlation between cTnl and NT-proBNP levels in patients with congenital cardiac malformations: cTnl tended to decrease with the increase in NT-proBNP.

Conclusions: cTnl has increased values in the first 24 postoperative hours in children with congenital cardiac malformations. There is a positive correlation between cTnl and NT-proBNP values suggesting the association of an early postoperative myocardial dysfunction in children with heart surgery.

\section{QUANTIFIED EVALUATION OF THE DUCTUS ARTERIOSUS}

\author{
A. Lakkundi ${ }^{1}$, K. de Waal ${ }^{1}$, I. Zonnenberg ${ }^{2}$
}

${ }^{1}$ Neonatology, John Hunter Hospital, Newcastle, NSW, Australia, ${ }^{2}$ Neonatology, VU Medical Centre, Amsterdam, The Netherlands

Introduction: We evaluated and quantified all ductal scans requested for predefined clinical signs and determined associations between clinical signs, ultrasound parameters and hemodynamic significance (HSDA) defined as ductal diameter (DAd) $>1.5$ or $>2.0 \mathrm{~mm}$.

Methods: Clinical signs were scored as mild (murmur, wide pulse pressure, hyperactive precordium), respiratory (difficulties to wean from or increase in respiratory support) or serious (hypotension, pulmonary edema or haemorrhage, congestive heart failure). Scans included central blood flow, DAd, ductal maximum velocity (Vmax), left pulmonary artery diastolic flow velocity (LPAd), $\mathrm{LA} /$ Ao ratio and flow pattern in the descending Aorta (DAo).

Results: Eighty scans in 48 patients were analysed. Median (range) gestational age was 26 (23-31) weeks, postnatal age 7 (1-60) days. No associations were found between clinical signs and DAd $>1.5$ or $2.0 \mathrm{~mm}$. A murmur was best correlated with Vmax (Pearson 0.52, p 0.000). Hypotension and pulmonary haemorrhage were correlated with a larger ductal diameter (Pearson 0.34, p 0.002 and $0.40, p 0.000)$. A DAd $>1.5 \mathrm{~mm}$ was associated with higher LPAd, LA/Ao ratio and LVO (mean difference $15 \mathrm{~cm} / \mathrm{s} ; 95 \% \mathrm{Cl} 8$ to $23,0.24 ; 95 \% \mathrm{Cl} 0.12$ to 0.36 , $93 \mathrm{ml} / \mathrm{kg} / \mathrm{min} ; 95 \% \mathrm{Cl} 30$ to 156 respectively). A DAd $>2.0 \mathrm{~mm}$ was associated with a lower Vmax (mean difference $-95 \mathrm{~cm} / \mathrm{s} ; 95 \% \mathrm{Cl}-53$ to -136 ) and more cases with reverse flow in the DAo (5\% vs $55 \%$ ).

Conclusion: Clinical signs cannot predict ductal size. ADAd $>1.5$ is associated with known ultrasound parameters, a DAd $>2.0$ only with Vmax and DAo pattern. 